\title{
DETECTING BAD DESIGN AND BIAS FROM PATENTS
}

\author{
Melluso, Nicola (1,2); \\ Pardelli, Sara (3); \\ Fantoni, Gualtiero $(1,2,3)$; \\ Chiarello, Filippo $(1,2,3)$; \\ Bonaccorsi, Andrea (1,2,3) \\ 1: University of Pisa; \\ 2: B4DS Lab - Business Engineering for Data Science; \\ 3: Erre Quadro s.r.l.
}

\section{ABSTRACT}

The representation of the product use context is a well established design practice in Engineering Design. Recently, design theory is studying the product interaction involving several cognitive aspects such as the possible conditions in which a wrong interaction occurs. The aim of this paper is to find a quantitative evidence of the causes of these misuses. In particular, this study focuses on the detection of bad design and biases.

In this paper, we propose a method that helps to the automatic detection of bad design and biases from patents. The method is based on an approach that defines syntactic rules to detect sentences containing these artifacts. These rules are defined based on an exploratory analysis of the explicit mention of "bad design" and "bias" and then, tested with multiple experiments on a sample of patents. The results give a first quantitative evidence of the presence of bad design and biases in patents and consequently of their importance in the design theory. In particular, it is provided a fine grain analysis of the linguistic structure of sentences containing these artifacts helping designers in detecting automatically them from patents.

Keywords: Design cognition, Design theory, Semantic data processing, Bad Design, Bias

\section{Contact:}

Melluso, Nicola

University of Pisa

Department of Civil and Industrial Engineering

Italy

nicolamelluso@gmail.com

Cite this article: Melluso, N., Pardelli, S., Fantoni, G., Chiarello, F., Bonaccorsi, A. (2021) 'Detecting Bad Design and Bias from Patents', in Proceedings of the International Conference on Engineering Design (ICED21), Gothenburg, Sweden, 16-20 August 2021. DOI:10.1017/pds.2021.117 


\section{INTRODUCTION}

Many theoretical models and frameworks have been proposed in Engineering Design (ED) over the years. Some focus more on the technical aspects, others on the interaction with the user; some are very prescriptive and analytic while others try to stimulate intuitive reasoning, and so on. All of these models partially address particular aspects such as the user's role, values and needs (Gero et al. 2002). However, ED still lacks studies that take into account explicit representation of failures or the cognitive aspects that involve a product design. In fact, a good design practice uses artifacts to formalize the product use context.

Although the role of cognitive aspects in ED is well established, few works are working on expanding and discussing the possible identification of new cognitive artifacts, in addition to the well-known affordances. For example, recent developments in Cognitive Psychology (Kahneman 2011) allow us to discuss in more depth the product use context.

In this paper we attempt to make a quantitative contribution to the exploration of two cognitive artifacts: bad design and biases. This study has a twofold objective. First, it aims at demonstrating the presence of bad design and biases in technical documents, i.e. patents. Second, it provides a methodology that uses an automatic approach to the detection of such artifacts in the text.

The paper is structured as follows. Section 2 discusses the theoretical background of ED related to bad design (Section 2.1), biases (Section 2.2) and the extraction of design relevant information from text (Section 2.3). Section 2 and Section 3 describes the methodology used to detect respectively bad design and biases. Finally, Section 4 discusses the conclusions and the future developments.

\section{BACKGROUND}

In this section we discuss the literature that is relevant to our work. In Section 2.1 and Section 2.2 we define respectively the concepts of bad design and bias showing its relevant scientific background; in Section 2.3 we review the literature related to the extraction of design concepts from text.

\subsection{Bad Design}

Bad design is not explicitly defined in literature. Even if it is a concept widely accepted in the nonacademic literature (white papers, patents, books etc.) (Hay et al. 2020), it is possible to find its link to the engineering design field. (Norman 1988) first mentioned the concept of bad design ("Human error? No, bad design"). With his definition, Norman intends to attribute to the designer the responsibility of a product that does not match the expected behaviour from the user point of view.

However we can link the concept of bad design with existing literature about the design perspective of the FBS framework (Gero and Kannengiesser 2002). In particular, bad design is linked to the notion of affordance. We define the affordances as the "possible actions of a product" (Gibson 1966) or "the set of all potential human behaviors that a product might allow" (Gibson 2014). Affordances can be recognized from experience, can be learned and also inferred by analogy. Gaver (1991) states that affordances are context dependent manipulation possibilities from the point of view of a particular actor. The actor is considered to be the entity, human or otherwise, capable of taking action. From the design perspective of the extended model of the FBS, introduced by Cascini et al. (2011), the concept of affordance is strictly related to the notion of misuse. Misuses are those conditions in which the user manipulates the product in ways that were not intended by the designer, still keeping the same goal. It is proposed to distinguish between two kinds of misuses. The first case occurs when the user's manipulation is based on her/his belief that the product affords a behaviour, but that behaviour was not intended by the designer (Spreafico et al. 2015). The second case occurs when the user and designer agree on the affordances, but the user has erroneous expectations about the product's behaviour (Cascini et al. 2013). Summing up, the misuses are the possible behaviors (interpreted by the user as possibilities of achieving goals) of the system, coming from its structure and linked to the goals the product was designed for (Filippi et al. 2013). These cases of misuses perfectly describe the occurrence of a bad design.

Hence, a bad design occurs when the product does not meet the expected behaviour from the user's point of view.

The presence of badly designed products in everyday life is due to the fact that the design process does not have an optimal solution: designers can not always match the user's needs. Even if a large 
literature in cognitive psychology and engineering design focuses on the right interpretation of artifacts, there is still a lot to be done to make the concept of bad design global and operational (Chiarello, et al. 2019). In particular, what is needed is an effort to develop a quantitative methodology to rapidly identify such artifacts. In particular, an automatic retrieval method of extracting artifacts, such as bad design, could allow several improvements in the design processes, such as: (i) the understanding of which products are most affected by bad design; (ii) the detection of problems that afflict a product following a bad design or (iii) the extraction of consequences that bad design problems have on users.

\subsection{Bias and Engineering Design}

The concept of cognitive bias belongs to one of the most influential research advancements in social science (Kahneman et al. 1982). According to this paradigm, the ability of human subjects to perform cognitive tasks is subject to limitations that originate from memory capacity and the nature of information processing . People do not perform exhaustive search of alternatives, but rather use wellfunctioning, rules of thumb (heuristics), and are subject to systematic distortions (biases) in their decisions (Kahneman 2011).

Recent developments in Engineering Design theory made available tools for the construction of graphical friendly representations of abstract functions (functional maps) that help to identify and mitigate biases in product design and development. For example, the theoretical foundations of Functional Analysis (Gabelloni et al. 2011; Bonaccorsi et al. 2011; Cascini et al. 2013) have been recently applied with success to map, from a functional perspective, the designer and user activities in approaching the product artifacts.

However, given the importance of biases in engineering design activities, the identification of such cognitive distortions is not a trivial task. State of the art literature proceeds in this activity using qualitative and quantitative approaches. Although very effective in certain fields, such as Behavioral Economy and Psychology, Engineering Design still lacks systematic methods to perform this task (Bonaccorsi et al. 2020). This leads us to underline the twofold aim for this paper.

First, all the concepts that relate to a cognitive distortion or a kind of systematic deviation in judgement does not always refer to the name of "bias" (Toh et al. 2016). There are certain fields that can express this concept differently. For example, the first appearance of the word "bias" in engineering design came as a synonym for "ambiguous design situations" (Otto and Antonsson 1991). The ambiguity in naming this phenomenon leads to the need for defining a methodology that aims to extract all the possible manifestations of it in this field.

Second, the occurrence of any sort of cognitive distortion is at the base of product failures (Bonaccorsi et al. 2020). An effective task of bias reduction in all the activities involved in engineering design produces a wide range of positive effects. In order to reduce all the bias effects it is crucial to have a practical methodology that allows designers to identify them. In this paper we aim to give a first methodological perspective to detect them from patents.

\subsection{Extracting technical design knowledge from text}

The extraction of design relevant information from technical texts is a task that is affecting latest research endeavours in different academic fields, such as Engineering Design and Artificial Intelligence. The work done by the academic community in using text mining for engineering design purposes is currently demonstrating promising results (Chiarello et al. 2019a; Chiarello et al. 2020; Fantoni et al. 2020). For example, NLP-based methodologies are used to detect and extract information about the functions, the physical behaviours and the states of the system directly from the text of a patent in an automatic way (Fantoni et al. 2013). These works suggest that patents are the most suitable source of information for such tasks (Chiarello et al. 2018). Patents are publicly available and designers (Bonaccorsi et al. 2007), use them as a primary source of technical information.

In this paper we focus on technical knowledge aimed at supporting the design process, from the conceptual stage down to industrial development (Chaudhari et al. 2020). Design knowledge is tacit and embedded in most cases and it is difficult for designers to express their knowledge fully and explicitly. For this reason, a set of Knowledge Acquisition processes (Wu et al. 2018) are adopted by researchers with several methodological perspectives (from established engineering disciplines to psychology, from ethnographic to simulation and operations research) and use both qualitative and 
quantitative techniques (Kan et al. 2009; Shealy et al. 2019). Thanks to the increasing availability of data, text mining (TM) has proven to be an important approach used by designer due to the possibility to: (i) extract information that are relevant for the design process but that are hidden in massive quantity of unstructured documents (Chiarello et al. 2018; Chiarello et al. 2019b); (ii) exploit publicly available sources, thus helping in resolving the problem of the non-availability of data (Parraguez et al. 2017).

However, text mining still lacks techniques able to overcome semantic issues due to the hidden nature of information in patents, considering that this information is rare (for legal reasons it is not mandatory to explicitly insert all the technical relevant information) and fuzzy (applicants use a linguistic form hard to comprehend because the try to make hard to find the patent or the design around it).

The work of Chiarello et al. (2019) is a first attempt of using TM techniques to extract artifacts that are able to activate spontaneous and immediate users' reactions, such as affordances. They propose a set of three different approaches of NLP techniques to extract meaningful affordance information from the full text of patents: 1) a simple word search, 2) a lexicon of affordances and 3) a rule-based system. This proven ability of automatic extraction of affordance leads us to consider the possibility of further performing an automatic extraction of other design-relevant knowledge, included in artifacts such as bad design and bias.

\section{BAD DESIGN DETECTION}

In this section we show the approach used to detect bad design from patents. The detection of textual representations of design artifacts, usually approached with Natural Language Processing (NLP) techniques (Chiarello et al. 2018; Chiarello et al. 2019), is not a trivial task. This is due to the "hidden" nature of such entities (see Section 2.3). The main problem to tackle is that a bad design could be expressed in different ways. For this reason, this study aims at defining some cues that help to perform the detection of bad design in its various ways of expression.

The following subsections describe our approach: we performed a primary search of the sentences containing bad design in patents (Section 3.1), we formulated rules for the automatic extraction (Section 3.2) and we tested them in terms of precision, recall and F-score (Section 3.3).

\subsection{Text search of "bad design"}

For the first task, we built a query that searches in the Title, Abstract and Claims of patents the word "bad design" and its synonyms, such as lexical variations of the negative adjectives ("poor", "wrongly", "unpleasant", "inadequate" etc...). This query has been performed over the Erre Quadro S.r.l.1 database. This database is a proprietary database containing over 90 million patents from the DOCDB and European Patent Office (EPO) repositories.

The results of this search give us a preliminary metric of how hard it is to find this artifact in patents. Table 1 shows the distribution of the term within the International Patent Classification (the first 10 results are shown in decreasing order of occurrences of the term "bad design"). This table allows us to formulate the following observations: (i) within the first 10 sub-classes, the class G06 (Computing; Calculating; Counting) occurs four times and the section G (Physics) seven times; (ii) the term bad design appears very rarely, less than one in thousand cases; (iii) it is not equally distributed across patent classes; (iv) there is a strong correlation between the primary search of "bad design" and the text-search of "affordance" in the previous study of Chiarello (2019).

\subsection{Rules to detect Bad Design}

The search for the explicit form of "bad design" showed poor results in terms of frequency. However, its distribution among the IPC classes suggests an exploratory analysis only of the classes that explicitly declare a bad design with higher frequency. This analysis consisted of reading the Prior Art section of section that contain at least an occurrence of the expression "bad design". We choose to analyze the Prior Art because it is usually constituted by all information that has been made available in any form before a given date that might be relevant to a patent's claims of originality. In this case we analyzed the content of 500 patents belonging to IPC classes shown in Table 1. In particular, we

\footnotetext{
${ }^{1}$ https://www.errequadrosrl.com/
} 
analyzed a sample of 50 patents per class. Each sample consists of patents containing the word "bad design" plus a random set that aims to make the sample reach the size of 50. This task involved a panel of experts made of two PhD students in Smart Industry and two researchers with academic backgrounds on Engineering Design. This exploration led to a first crucial observation: a design problem is often declared due to an overabundance of features (artifacts). This observation finds scientific consensus from the insights provided by Cascini (2011) and from foundations of the axiomatic design method.. The overabundance of features could be perceived due to the following causes: features size (the features exceed or fail the expectations in terms of size), features number (the number of features exceeds or fails the expectations) and features location (the feature is wrongly located on the product). Hence, we can formulate the following hypothesis: the overabundance of features leads to misuses, and consequently to a bad design. This hypothesis suggests that it could be possible to define some lexical rules that help to detect bad design from text. In particular, we could be able to find sentences that refer to a bad design.

Table 1: Distribution of the term "bad design" in top 5 IPC subclasses.

\begin{tabular}{|l|l|l|l|}
\hline Class name & $\begin{array}{l}\text { IPC } \\
\text { Class }\end{array}$ & $\begin{array}{l}\text { All } \\
\text { patents }\end{array}$ & $\begin{array}{l}\text { Patents including } \\
\text { “bad design" }\end{array}$ \\
\hline Electric digital data processing & G06F & 5.251 .009 & 210 \\
\hline Transmission of digital information & H04L & 2.724 .700 & 55 \\
\hline Data processing system or methods & G06Q & 1.250 .239 & 24 \\
\hline $\begin{array}{l}\text { Computer systems based on computational } \\
\text { models }\end{array}$ & G06N & 131.419 & 22 \\
\hline Semiconductor devices & H01L & 4.546 .970 & 16 \\
\hline Wireless communication networks & H04W & 1.564 .916 & 15 \\
\hline Measuring electric variables & G01R & 1.123 .341 & 14 \\
\hline Image data processing or generation & G06T & 931.031 & 12 \\
\hline Information storage based on relative movement & G11B & 1.441 .476 & 10 \\
\hline
\end{tabular}

A text that describes the prior art of a patent can express an overabundance of features using different expressions. However, a reader may note that the way such problems are presented may follow recurring language patterns. Consequently, we can summarise the cases in which such expressions may appear in the text with the linguistic rules described in Table 2.

Table 2: Linguistic rules to detect "bad design"

\begin{tabular}{|l|l|l|}
\hline Rule Name & Description & Example terms \\
\hline R1 & $\begin{array}{l}\text { The sentence starts with } \\
\text { expressions that introduce a } \\
\text { statement that refers, usually to } \\
\text { contradict or agree, something that } \\
\text { has been said previously }\end{array}$ & $\begin{array}{l}\text { however, if, for } \\
\text { example, when, } \\
\text { although, ... }\end{array}$ \\
\hline R2 & $\begin{array}{l}\text { The sentence contains terms that } \\
\text { describe the need for much effort } \\
\text { or skill to accomplish, deal with, or } \\
\text { understand }\end{array}$ & $\begin{array}{l}\text { difficult, wrong, } \\
\text { mistakenly, } \\
\text { inconvenient, } \\
\text { lumbersome, too } \\
\text { much, too many, too } \\
\text { small, not enough, ... }\end{array}$ \\
\hline R3 & $\begin{array}{l}\text { The sentence mention the feature of } \\
\text { the product }\end{array}$ & $\begin{array}{l}\text { button, key, pointer, } \\
\text { bundle, hand, ... }\end{array}$ \\
\hline
\end{tabular}

Let us show an example to better explain the formulation of these rules. These are three extracts from three patents describing the bad design related to the feature "button":

The bigger is the button the higher is the probability of distraction. For example, a button bigger than the display leads to a wrong use of the device. 
Too many buttons may confuse the user. If the number of buttons increases too much, a user may have difficulty in choosing the right one.

A button disposed symmetrically to the display creates difficulties in the manipulation of the machine.

These three examples refer respectively to a problem of features size, features number and features location. In the first example, the second sentence expresses the bad design using an introductory expression ("for example"), citing the feature ("button") and using the term "wrong"; these correspond to the three rules defined in Table 2. In the second example, the second sentence expresses a bad design using the same three rules. In the third example, the sentence expresses a bad design citing the feature ("button") and using the term "difficulties". However, these rules can not be considered separately. In particular, given the large use of terms referring to the feature of the product, the sole satisfaction of the R3 it is not sufficient to state that the sentence contains a bad design. Therefore, as shown in the next section, the R3 does not work alone. It resulted to be useful to increase the precision of the detection if it co-occurs with R1 and R2.

\subsection{Bad Design detection test}

The definition of rules to detect bad design from text requires a test that asserts how correctly the method works. The test has been performed using the spacy (Honnibal et al. 2017) Entity Matcher, an open-source software library for Natural Language Processing, written in Python and Cython. We developed a system that extracts sentences containing bad design using the rules described in Section 3.2. We tested the system performing an experimental detection of sentences over the "Background" section of 10,000 patents. In particular, we used a random sample of 5,000 patents belonging to the G06F patent class and a random sample of 5,000 belonging to the H04L patent class.

We evaluate the performance of the detection system performing multiple experiments: we run 7 different experiments that represent all the combinations of the rules. For each experiment we measure the accuracy of the detection in terms of precision, recall and F-score. We employed our panel of experts (two PhD students and two researchers) to evaluate the results of the detection. They checked if the sentences detected were relevant or not and, in turn, if the patent containing the sentence was relevant. A relevant sentence is the one that correctly contains a bad design. As a consequence, a relevant patent is a patent that contains at least one relevant sentence. The sentence precision is calculated as the fraction of relevant sentences among the extracted sentences, while the patent precision is calculated as the fraction of relevant patents among the sample patent set. The estimated recall is calculated taking into account an estimation based on the formula (1), due to the fact that there is no ground truth for the bad design artifact.

$$
\text { estimated recall }=\frac{n . o f \text { relevant instances }}{\text { total n.of unique relevant instances }}
$$

Then. of relevant instances is the number of relevant sentences (or relevant patents) detected in the experiment. The total $n$. of unique relevant instances represents all the relevant sentences (or relevant patents) detected by all the experiments (namely in the whole sample set). The F-score combines precision and recall with the harmonic mean of the two values. Figure 1 summarizes the resulting measures for the different combinations of rules. This Figure suggests that the single rule with the highest accuracy is R2. Even though the combination of the three rules leads to the highest probability of finding a bad design (highest precision values) it is rare to find those sentences that contain all three elements. The experiments R1 + R2 and R2 + R3 show high precision but low recall. This confirms that even though the combination of these rules is effective, they hardly appear together in patents. Table 3 shows a sample of sentences extracted from the experiments that satisfy all the three rules. These experimental tests lead us to significant results: we detected a total number of 5,722 unique relevant sentences among a total number of 183,186 sentences contained in the experimental patent set, and a total number of 2,218 relevant patents among a sample of 10,000. This means that about $22 \%$ of patents could contain the description of a bad design. Such analysis provides a first quantitative evidence of the presence and importance of this engineering design artifact. 


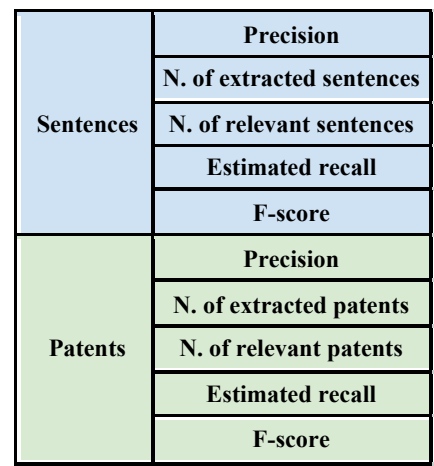

\begin{tabular}{|c|c|c|}
\hline $\mathbf{R}_{\mathbf{1}}$ & $\mathbf{R}_{\mathbf{2}}$ & $\mathbf{R}_{\mathbf{3}}$ \\
\hline 0.11 & 0.51 & 0.03 \\
\hline 33,300 & 5,839 & 12,485 \\
\hline 3,663 & 2,977 & 374 \\
\hline 0.64 & 0.52 & 0.07 \\
\hline 0.19 & 0.52 & 0.04 \\
\hline 0.10 & 0.43 & 0.03 \\
\hline 8,159 & 3,337 & 3,496 \\
\hline 816 & 1,435 & 105 \\
\hline 0.37 & 0.65 & 0.05 \\
\hline 0.16 & 0.52 & 0.04 \\
\hline
\end{tabular}

\begin{tabular}{|c|c|c|}
\hline $\mathbf{R}_{\mathbf{1}}+\mathbf{R}_{\mathbf{2}}$ & $\mathbf{R}_{\mathbf{2}}+\mathbf{R}_{\mathbf{3}}$ & $\mathbf{R}_{\mathbf{1}}+\mathbf{R}_{\mathbf{3}}$ \\
\hline 0.64 & 0.69 & 0.04 \\
\hline 490 & 824 & 5,203 \\
\hline 314 & 569 & 208 \\
\hline 0.05 & 0.10 & 0.04 \\
\hline 0.10 & 0.17 & 0.04 \\
\hline 0.59 & 0.58 & 0.02 \\
\hline 448 & 694 & 3,210 \\
\hline 262 & 403 & 79 \\
\hline 0.12 & 0.18 & 0.04 \\
\hline 0.20 & 0.28 & 0.03 \\
\hline
\end{tabular}

\begin{tabular}{|c|}
\hline $\mathbf{R}_{\mathbf{1}}+\mathbf{R}_{\mathbf{2}}+\mathbf{3}_{\mathbf{2}}$ \\
\hline 0.90 \\
\hline 77 \\
\hline 69 \\
\hline 0.01 \\
\hline 0.02 \\
\hline 0.89 \\
\hline 76 \\
\hline 68 \\
\hline 0.03 \\
\hline 0.06 \\
\hline
\end{tabular}

Figure 1: Results of the detection of bad design

Table 3: Sentences with a high probability to contain bad design.

\begin{tabular}{|l|l|}
\hline Sentence & Rules satisfied \\
\hline $\begin{array}{l}\text { In addition, the user must move the pointer subtly, } \\
\text { for example, to position the pointer on a small icon, } \\
\text { but it is difficult to perform such manipulations using } \\
\text { commands such as those described above. }\end{array}$ & $\begin{array}{l}\text { R1 (In addition) } \\
\text { R2 (difficult to) } \\
\text { R3 (pointer) }\end{array}$ \\
\hline $\begin{array}{l}\text { Such an apvnplication presents difficulties for } \\
\text { conventional eye tracking apparatuses because of the } \\
\text { disparate focus requirements for the eye and the scene } \\
\text { as viewed through the secondary optical apparatus. }\end{array}$ & R1 (Such) \\
R2 (difficulties) \\
\hline $\begin{array}{l}\text { As the broadcasting system becomes more advanced } \\
\text { and complicated, more experienced and professional } \\
\text { technicians are required to manage the system. }\end{array}$ & R1 (As) \\
\hline R2 (complicated) \\
\hline $\begin{array}{l}\text { However, the function keys with individual functions } \\
\text { disposed in one keyboard may cause some users to } \\
\text { easily confuse one function with other ones. }\end{array}$ & R1 (However) \\
\hline $\begin{array}{l}\text { If the keys become too numerous, some of the keys } \\
\text { may be difficult to reach, and users may have } \\
\text { difficulty remembering the purposes of all keys. }\end{array}$ & $\begin{array}{l}\text { R1 (If) } \\
\text { R2 (difficult) } \\
\text { R3 (keys) }\end{array}$ \\
\hline
\end{tabular}

\section{BIAS DETECTION}

As said before (see Section 2.1), bad design could introduce misuses, false affordances or any other wrong behaviour that causes a mistaken manipulation. One of these effects could be related to a cognitive distortion occurring in the product context use. This is the case of the appearance of a bias. This section aims to describe the approach used to define a preliminary methodology to automatically detect bias from text. In particular, we used the same three-steps approach of the bad design detection (Section 3): the text-search in Section 4.1, the definition of rules in Section 4.2 and the discussion of the experimental test in Section 4.3.

\subsection{Text search for "bias"}

A preliminary text-search of the term "Bias" has been performed over the Title, Abstract and Claims of the patent database (see Section 3.1). As we can see in Table 4, also the term "bias" is rarely used in patents.

However, the distribution of the term within the IPC classes is slightly different from the one of "bad design" and the one of "affordance" (Chiarello et al. 2019), in terms of frequency and variety. First, the term "bias" appears more frequently than the term "bad design". Second, the word "bias" also appears in classes not related to Engineering Design. This is a preliminary confirmation of the 
interdisciplinary nature and wide applicability of this concept discussed in the Background section (see section 2.2.). As for the bad design extraction, this first preliminary text-search has been the starting point for observing the patent dataset and to defining lexical rules that improve the identification of this entity in patents.

Table 4: Distribution of the term "bad design" in top 5 IPC subclasses.

\begin{tabular}{|l|l|l|l|}
\hline Class name & IPC & All Patents & Patents including "bias" \\
\hline Preparations for medical purposes & A61K & $5,348,390$ & 3,621 \\
\hline Electric digital data processing & G06F & $5,251,009$ & 2,266 \\
\hline Microorganisms or enzymes & C12N & $1,656,545$ & 1,922 \\
\hline Diagnosis & A61B & $2,027,515$ & 1,773 \\
\hline Therapeutic activity of chemical compounds & A61P & $3,192,620$ & 1,656 \\
\hline Measuring or testing processes involving enzymes & C12Q & 739,242 & 1,431 \\
\hline Investigating or analysing materials & G01N & $2,953,100$ & 1,352 \\
\hline Data processing systems or methods & G06Q & $1,250,239$ & 899 \\
\hline
\end{tabular}

\subsection{Rules to detect Bias}

The approach used for performing an automatic detection of biases follows the same steps of the one used for detecting bad design. In fact, an exploratory analysis of the patent set identified in the first step (see Section 4.1) led to the identification of some regularities that help to perform this task. We choose to involve our panel of experts in an exploratory analysis. Since the term bias is affected by different meanings related to several non-engineering domains, we approached exploratory analysis in the following manner: we analysed a sample of 500 patents, made of sets of 100 documents randomly selected for IPC classes among the patents containing the term "bias" and that are related to the engineering domain. We select the top 5 classes of patents in which also the word "bad design" appears. This analysis led to the identification of the following hypotheses.

First, a cognitive related expression often occurs in the description of a bias. A cognitive related expression is a term, usually a verb, that refers to an aspect of the intellectual functions and processes (such as attention, memory, judgement, evaluation, reasoning etc...). In practice, a bias is described in those sentences that contain a set of terms that implicitly or explicitly suggests a cognitive process. Cognitive verbs (such as "believe", "assume", "conclude" etc.) are those expressions that frequently appear when a bias is described.

Second, a negative adverb often occurs in the description of a bias. A positive adverb, such as "correctly", expresses "a way that is in agreement with the true facts or with what is generally accepted"2. This definition leads us to state that if a positive cognitive adverb accepts what is generally accepted, a negative cognitive adverb, such as "wrongly" or "mistakenly", disagrees with the true facts.

However, these two hypotheses can not be considered separately. Given the large use of cognitive adverbs and cognitive verbs in the patent language, the sole presence of one of these elements in a sentence it is not sufficient to state that the sentence contains a bias. For this reason, we formalize these hypotheses defining only the following lexical rule: if a sentence contains both a cognitive adverb and a cognitive verb it will describe a bias.

\subsection{Bias detection test}

The experimental test performed to validate the rules for the detection of biases followed the same approach used for the detection of bad design. In particular, we developed an Entity Matcher system performing the detection test over the sample of 10,000 patents described in Section 3.3.

In this case, the measure of the accuracy of this test could only be calculated in terms of precision. As Bonaccorsi et al. (2020) state, the recall for this concept is difficult to measure due to the absence of a ground truth.

\footnotetext{
${ }^{2}$ https://dictionary.cambridge.org/en/correctly
} 


\begin{tabular}{|l|}
\hline Sentence \\
\hline $\begin{array}{l}\text { That is, many consumers may falsely believe that the charger will not consume power } \\
\text { unless it is actually connected to the electrical device and being used for charging purposes, } \\
\text { even when it is plugged into an outlet. }\end{array}$ \\
\hline $\begin{array}{l}\text { Thus, the recipient may be confused regarding why he or she has been denied access, and } \\
\text { the sender may wrongly conclude that the recipient has received access to the file. }\end{array}$ \\
\hline $\begin{array}{l}\text { Users may improperly assume that they have entered improper data, improperly operated } \\
\text { the application, and/or conclude the application is programmatically flawed. }\end{array}$ \\
\hline $\begin{array}{l}\text { Upon falling asleep, he may unconsciously assume that supine position out of habit- } \\
\text { increasing the adverse conditions of sleep apnea or snoring }\end{array}$ \\
\hline
\end{tabular}

Our panel of experts assert that 847 sentences, out of 951, correctly contain a bias. Hence, the rule detects biases with $89 \%$ accuracy. Table 5 shows some of these sentences where in bold are highlighted the lexical elements that suggest the presence of a bias. Despite the high precision, the number of sentences detected are very few compared to the total number of sentences contained in the sample set $(183,186)$. This highlights the difficulty of detecting biases, even though their presence is confirmed, as shown in Table 5. As supposed before a bad design misleads the user and introduces a series of effects: distraction, tiredness, cognitive biases and interaction mistakes. This is confirmed by the sentences shown in Table 5: bad design is co-mentioned with biases (i.e. "the radio may be distracting to a driver"). Therefore all of these concepts could be analysed together in a wider perspective which may include the detection of their relationships.

\section{CONCLUSIONS AND FUTURE DEVELOPMENTS}

This paper offers a preliminary discussion of the representation of cognitive failures in a product use context. In particular, it analyzes two cognitive artifacts: bad design and bias. The analysis has been conducted using a linguistic approach that aimed to detect such entities from patents. The results of this analysis provide the following contributions. First, it has been demonstrated that bad design is an artifact that appears in patents. Even though it is not explicitly declared with the expression "bad design", it is contained implicitly in some sentences. These sentences show a regular pattern of lexical rules that would simplify their detection, at least in patents. Second, the methodology used to detect bad design has been tested successfully. The experiments suggest that a deeper semantic analysis could lead to the development of an automatic detection system. Third, even though biases are more explicitly declared in patents, their detection is still difficult. The definition of only one rule suggests that biases do not follow recurrent lexical patterns. In addition, despite the high precision of the experiment test, the low number of sentences detected further confirm the difficulty of capturing such an entity. Finally, the methodology used in this study could be adopted in future design practices. Given the existence of these artifacts, a systematic approach for detecting sentences that contain bad design or bias could help a designer in improving his product design activities.

However, there is a room for improvement. An important limitation of this paper regards the need to distinguish the approaches to detect bad design and bias according to the source. For example, the presence of the overabundance of features is a characteristic of bad design that is typical for patents. This is due to the fact that, for example, patents miss the descriptions of design activities, because they are not relevant for "patentability". Consequently, there would be other causes related to bad design that need further exploration in order to make the analysis more comprehensive. This is the key issue pointed out by Lee et al. (2018) and Georgiev and Georgiev (2018). Bad design could be the consequence of an erroneous execution of a phase of the overall product development process and it could be due to a lack of knowledge of the designer itself. These two causes are rarely reported in patents and consequently, it is difficult to detect them. This is a limitation of our work that needs to be addressed in the future.

\section{ACKNOWLEDGEMENTS}

We would like to thank Ilenia Cirri and Erre Quadro Srl for the precious help in the analysis. 


\section{REFERENCES}

Apreda, R., Bonaccorsi A., Fantoni G. and Gabelloni G. (2014). "Functions and Failures: How to Manage Technological Promises for Societal Challenges.” Technology Analysis \& Strategic Management

Bonaccorsi, A. (2011). "A Functional Theory of Technology and Technological Change."

Bonaccorsi A., Apreda R., and Fantoni G. (2020). "Expert Biases in Technology Foresight. Why They Are a Problem and How to Mitigate Them." Technological Forecasting and Social Change 151: 119855.

Bonaccorsi, A. and Fantoni, G. (2007), “Expanding the functional ontology in conceptual design”, International Conference on Engineering Design (ICED07).

Cascini, G., Del Frate, L., Fantoni, G. and Montagna, F. (2011), “Beyond the design perspective of Gero's FBS framework", In: Design computing and cognition, Springer, Vol. 10, pp. 77-96.

Cascini, G., Fantoni, G., and Montagna, F. (2013). "Situating Needs and Requirements in the FBS Framework." Design Studies 34 (5): 636-62.

Chaudhari, A. M., Bilionis I., and Panchal J. H. (2020). "Descriptive Models of Sequential Decisions in Engineering Design: An Experimental Study.” Journal of Mechanical Design 142 (8)

Chiarello, F., Cimino A., Fantoni G., and Dell'Orletta F. (2018). "Automatic Users Extraction from Patents." World Patent Information 54 (September): 28-38.

Chiarello, F., Cirri I., Melluso I., Fantoni G., Bonaccorsi A., and Pavanello T.. (2019a). "Approaches to Automatically Extract Affordances from Patents.” International Conference on Engineering Design (ICED19)1 (1): 2487-96.

Chiarello, F., Melluso N., Bonaccorsi A., and Fantoni G. (2019b). “A Text Mining Based Map of Engineering Design: Topics and Their Trajectories Over Time.” International Conference on Engineering Design 1 (1): 2765-74.

Chiarello, F., Bonaccorsi, A., and Fantoni, G. (2020). Technical Sentiment Analysis. Measuring Advantages and Drawbacks of New Products Using Social Media. Computers in Industry, 123, 103299.

Fantoni, G., Coli, E., Chiarello F., Apreda, R., Dell'Orletta, F. (2020), “Text mining tool for translating terms of contract into technical specifications: Development in the railway sector" Computers in Industry

Fantoni, G., Apreda R., Dell'Orletta F., and Monge M. (2013). "Automatic Extraction of Function-behaviourstate Information from Patents." Advanced Engineering Informatics 27 (3): 317-34.

Filippi, S., Barattin D. and Cascini G., (2013), “Analyzing the Cognitive Processes of an Interaction Design Method Using the FBS Framework.” International Conference on Engineering Design (ICED13)

Fischhoff, B., Slovic P., Lichtenstein S. (1978), "Fault Trees: Sensitivity of Estimated Failure Probabilities to Problem Representation.” Journal of Experimental Psychology. Human Perception and Performance 4 (2)

Gabelloni, D., Apreda R., Fantoni G. (2011), “On the Link between Features and Functions." In International Conference on Engineering Design (ICED 11) 15.-19.08. 2011, 1-12.

Gaver, W. W. (1991). "Technology Affordances." In Proceedings of the SIGCHI Conference on Human Factors in Computing Systems, 79-84. CHI '91. New York, NY, USA: Association for Computing Machinery.

Gero, J. S., and Kannengiesser U. (2002). "The Situated Function - Behaviour — Structure Framework." Artificial Intelligence in Design '02. https://doi.org/10.1007/978-94-017-0795-4_5.

Gibson, J. J. (2014), The Ecological Approach to Visual Perception: Classic Edition. Psychology Press.

Gibson, J. J. (1966), The Senses Considered as Perceptual Systems. MA: Houghton Mifflin

Kahneman, D. (2011), Thinking, Fast and Slow. Macmillan.

Hay L., Cash, P., and McKilligan, S. (2020). "The future of design cognition analysis". Design Science, 6, E20

Honnibal, M., and Montani, I. (2017). "spaCy 2: Natural language understanding with Bloom embeddings, convolutional neural networks and incremental parsing".

Kahneman, D., Slovic S.P., Slovic P., Tversky A. (1982), Judgment Under Uncertainty: Heuristics and Biases. Cambridge University Press.

Kan, J.W.T and Gero J.S. (2009), “A Generic Tool to Study Human Design Activities.” International Conference on Engineering Design (ICED09) 123-34.

Norman, D. A. (1988), The Psychology of Everyday Things. New York: Basic Books

Otto, K. N., and Antonsson E. K. (1991), “Trade-off Strategies in Engineering Design.” Research in Engineering Design 3 (2): 87-103.

Parraguez, P., Maier A. (2017), "Data-Driven Engineering Design Research: Opportunities Using Open Data." International Conference on Engineering Design (ICED17) 041-050.

Shealy, T., and Gero J.S. (2019), "The Neurocognition of Three Engineering Concept Generation Techniques." International Conference on Engineering Design (ICED19) 1833-42.

Spreafico, C., Fantoni G. and Russo D. (2015), "FBS Models: An Attempt at Reconciliation towards a Common Representation.” International Conference on Engineering Design (ICED15) 399-408

Toh, C. A., Strohmetz A.A. and Miller S.R. (2016), "The Effects of Gender and Idea Goodness on Ownership Bias in Engineering Design Education.” Journal of Mechanical Design 138 (10)

Wu, Z., Liao J., Song W., Mao H., Huang Z., and Mao H. (2018), "Semantic Hyper-Graph-Based Knowledge Representation Architecture for Complex Product Development.” Computers in Industry 100: 43-56. 